\title{
The impact of liver disease aetiology and the stages of hepatic fibrosis on the performance of non-invasive fibrosis biomarkers: an international study of 2411 cases
}

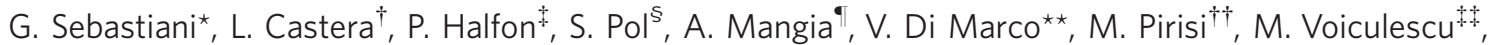 \\ M. Bourliere ${ }^{\S \S} \&$ A. Alberti ${ }^{*}$, 9
}

*VIMM-Venetian Institute of Molecular Medicine, Padova, Italy.

†Service d'Hépato-gastroentérologie,

Hôpital Haut Lévêque, C. H. U.

Bordeaux, Pessac, France

*Laboratoire Alphabio, Hospital

Ambroise Pare, Marseille, France.

sLiver Unit and Inserm U-567, Cochin Hospital, Paris, France.

"Division of Gastroenterology, Hospital Casa Sollievo della Sofferenza, IRCCS,

San Giovanni Rotondo, Italy.

${ }^{\star \star}$ Cattedra e Unità Operativa Comp-

lessa di Gastroenterologia ed Epatolo-

gia, University of Palermo, Palermo,

Italy.

†'Dipartimento di Medicina Clinica e Sperimentale, Università del Piemonte

Orientale, Novara, Italy.

*Department of Internal Medicine and

Nephrology, Fundeni Clinical Institute,

Bucharest, Romania.

§şHôpital Saint Joseph, Marseille,

France.

"Department of Histology, Microbiology and Medical Biotechnologies, University of Padova, Padova, Italy.

\section{Correspondence to:}

Prof A. Alberti, Venetian Institute for

Molecular Medicine (VIMM), Via Orus

2, 35100 Padova, Italy.

E-mail: alfredo.alberti@unipd.it

\section{Publication data}

Submitted 8 June 2011

First decision 26 June 2011

Resubmitted 3 August 2011

Accepted 1 September 2011

EV Pub Online 9 October 2011

\section{SUMMARY}

Background

Performance of non-invasive fibrosis biomarkers may be influenced by aetiology of chronic liver disease (CLD) and the stages of hepatic fibrosis, but large-scale studies are pending.

\section{Aim}

To investigate the effect of aetiogy and stages of hepatic fibrosis on the performance of fibrosis biomarkers.

\section{Methods}

A total of 2411 patients with compensated CLD (HCV $=75.1 \%$, $\mathrm{HBV}=10.5 \%, \quad \mathrm{NASH}=7.9 \%, \quad \mathrm{HIV} / \mathrm{HCV}=6.5 \%) \quad$ were consecutively enrolled in 9 centres. APRI, Forns'index, Lok index, AST-to-ALT ratio, Fib-4, platelets and Fibrotest-Fibrosure were tested against liver biopsy, considered the gold standard. The effect of the stages of hepatic fibrosis to diagnose significant fibrosis and cirrhosis ( $\geq \mathrm{F} 2$ and $\mathrm{F} 4$ respectively) was investigated through difference between advanced and non-advanced fibrosis stages (DANA). Performance was expressed as observed area under the ROC curve (ObAUROC) and AUROC adjusted for DANA (AdjAUROC).

\section{Results}

Performance of APRI and Fibrotest-Fibrosure was higher than other biomarkers. In all aetiologies, AdjAUROC was higher than ObAUROC. APRI showed its best performance in HCV monoinfected cases, with an AdjAUROC of 0.77 and 0.83 for $\geq \mathrm{F} 2$ and F4 respectively. In HBV and non-alcoholic steatohepatitis (NASH) patients, its performance was poor (AdjAUROC <0.70). Performance of Fibrotest-Fibrosure was good in all aetiologies for both $\geq F 2$ and $F 4$ (AdjAUROC $>0.73$ ), except for $\geq F 2$ in NASH (AdjAUROC $=0.64$ ). Performance of all biomarkers was reduced in HCV cases with normal ALT.

\section{Conclusions}

Aetiology is a major factor influencing the performance of liver fibrosis biomarkers. Even after correction for DANA, APRI and Fibrotest-Fibrosure exhibit the best performance. However, liver biopsy is not replaceable, especially to diagnose $\geq \mathrm{F} 2$ and in HCV carriers with normal ALT.

Aliment Pharmacol Ther 2011; 34: 1202-1216 


\section{INTRODUCTION}

Chronic infection with the hepatitis B (HBV) and C $(\mathrm{HCV})$ viruses and non-alcoholic steatohepatitis (NASH) are the main causes of chronic and progressive liver disease worldwide, leading to cirrhosis, end-stage liver disease and hepatocellular carcinoma (HCC). ${ }^{1-3}$ Moreover, due to common routes of transmission, up to one-third of patients infected with the human immunodeficiency virus (HIV) in the US and Europe are coinfected with HCV or HBV. ${ }^{4,5}$ These different aetiological forms of chronic liver disease (CLD) share a common histopathological pathway that is the formation and accumulation of fibrosis, which leads to progressive distortion of the hepatic architecture, that is the hallmark of evolution to cirrhosis. ${ }^{6}$ Natural history studies indicate that advanced fibrosis and cirrhosis develop in about $20-40 \%$ of patients with chronic hepatitis $\mathrm{B}(\mathrm{CHB})$ or chronic hepatitis $\mathrm{C}(\mathrm{CHC})$ and in a similar proportion of those with $\mathrm{NASH}^{7-9}$ Staging of liver fibrosis by liver biopsy represents the gold standard for prognostic assessment and, in $\mathrm{CHC}$ and $\mathrm{CHB}$, for deciding to initiate anti-viral therapy. ${ }^{10}$ Liver biopsy, however, has a number of limitations, being invasive, costly, difficult to standardise and disliked by many patients. ${ }^{11-13}$ Its universal use in CLDs is unpractical due to the huge number of patients who are often asymptomatic. ${ }^{2,14}$ Recently, increasing interest has been directed towards the use of non-invasive tools to assess liver fibrosis. These include simple markers based on routinary tests, like platelets, AST-toplatelet ratio index (APRI), Forns' index, Fib-4, Lok index and AST-to-ALT ratio (AAR), and other more sophisticated and patented tests, such as Fibrotest-Fibrosure. ${ }^{15-21}$ In the midst of a large series of publications on these markers, investigated mainly in $\mathrm{CHC}$, their implementation in clinical practice is still debated and large-scale studies in aetiological forms other than HCV are still limited. ${ }^{12,13,22}$ Recently, it has been shown that the performance of these biomarkers may be influenced by the prevalence of liver fibrosis stages defining advanced and non-advanced fibrosis in the analysed patients cohort. ${ }^{23,24}$ Poynard and colleagues have therefore concluded that area under the receiving operating characteristic curve (AUROC), that is generally used to describe performance, should be standardised accordingly. ${ }^{23}$ The aim of this large-scale, international, independent study was to investigate the effect of aetiology of liver disease and of the stages of hepatic fibrosis on the performance of several non-invasive biomarkers in patients with CLD.

\section{METHODS}

\section{Study design}

This was an International, multicentre retrospective study conducted in nine clinical centres across Europe. Patients with CLDs of any aetiology were enrolled, according to inclusion and exclusion criteria. Our aim was to investigate the effect of liver disease aetiology and the stages of hepatic fibrosis on the performance of a series of non-invasive biomarkers for liver fibrosis. Details of patients' demographics, aetiology of CLDs, laboratory results and liver biopsy were recorded in each Centre. The study was conducted according to the Declaration of Helsinki.

\section{Inclusion and exclusion criteria}

We included consecutive patients admitted between January 2003 and December 2008. The inclusion criteria were (i) a diagnosis of well-compensated CLD of any aetiology and (ii) availability of both liver biopsy and relevant parameters for the assessment of noninvasive biomarkers, performed on the same day. Exclusion criteria were (i) decompensated liver cirrhosis; (ii) comorbidities that could confound the interpretation of non-invasive biomarkers, including haemolysis, Gilbert's syndrome, thrombocytopenia not liver-related; (iii) history or evidence at entry of HCC; (iv) liver transplantation. All patients gave their informed consent to be included in the study. Out of 2805 consecutive patients with CLDs who had a liver biopsy and non-invasive biomarkers performed on the same day, we included 2411 patients. A total of 221 were excluded for decompensated liver cirrhosis, 56 for confounding factors for the non-invasive biomarkers, 105 for HCC and 14 for previous liver transplantation. Patients with chronic viral hepatitis were treatmentnaïve.

\section{Aetiologies of liver disease}

$\mathrm{HCV}$ infection was established by a positive third-generation anti-HCV test (Ortho Diagnostic Systems, Raritan, NJ, USA), with serum HCV-RNA detected by polymerase chain reaction (PCR) (Amplicor HCV Monitor test, Roche Diagnostics, Indianapolis, IN, USA). The HCV genotype was determined by INNOLipa (Innogenetics, Bayer, Ghent, Belgium). Diagnosis of HBV infection was based on the presence of HBsAg for more than 6 months, and $\mathrm{HBeAg} /$ anti-HBe were determined using commercial assays (Roche Diagnostics, Basel, Switzerland). HBV-DNA 


\section{G. Sebastiani et al.}

level was measured by real-time PCR. HIV infection was defined by HIV ELISA, confirmed by Western blot assay, with positive HIV-RNA. The diagnosis of NASH was based on liver histology. Secondary causes of steatohepatitis including significant alcohol consumption ( $\geq 100 \mathrm{~g} /$ week) and the use of hepatotoxic drugs were excluded. Among patients with either HCV or HBV infection, $487(20.2 \%)$ subjects admitted occasional alcohol consumption and $29(1.2 \%)$, moderate alcohol consumption.

\section{Histological assessment}

Liver biopsies were analysed in each centre by the local pathologist who was unaware of clinical data. Diagnosis of NASH was based on the common features including steatosis, inflammation (portal and lobular), hepatocyte ballooning and fibrosis, according to the scoring system recently developed by the NIHsponsored NASH Clinical Research Network. ${ }^{25}$ In patients with $\mathrm{CHC}$, or $\mathrm{CHB}$ or $\mathrm{HIV} / \mathrm{HCV}$ coinfection, fibrosis was scored according to METAVIR classification as follows: F0, no fibrosis; F1, portal fibrosis without septa; F2, portal fibrosis with few septa; F3, numerous septa without cirrhosis; and F4, cirrhosis. ${ }^{26-29}$ Staging of liver fibrosis in NASH patients was as follows: F0, normal connective tissue; F1, foci of perivenular or pericellular fibrosis in zone 3; F2, perivenular or pericellular fibrosis confined to zones 3 and 2, with or without portal/periportal fibrosis; F3, bridging or septal fibrosis; and F4, cirrhosis. ${ }^{30}$

\section{Non-invasive biomarkers for liver fibrosis}

The following non-invasive biomarkers were determined in all patients, according to published formulas: platelets, AAR, APRI, Forns' index, Lok index, Fib-4. FibrotestFibrosure values were obtained through Biopredictive (Biopredictive, Paris, France) or by courtesy of Prof. Thierry Poynard. For all non-invasive biomarkers investigated, the cut-off values indicated in the original reports or in subsequent studies in various aetiologies of CLD were applied. ${ }^{15-21,27,31-33}$

\section{Outcome measures}

Performance of non-invasive biomarkers was assessed for the detection of: (i) significant fibrosis ( $\geq \mathrm{F} 2$ according to METAVIR or NASH classification) and (ii) cirrhosis (F4) as these threshold are generally considered clinically relevant to initiate anti-viral therapy and for specific management and follow-up protocols. ${ }^{34-37}$

\section{Statistical analysis}

Descriptive results were expressed as mean \pm s.d. (standard deviation) or number (percentage) of patients with a condition. The $t$-test or non parametric Mann-Whitney test was used to compare quantitative data and the chisquared test was applied for comparison of frequency data. All tests were two-tailed and $P$-values $<0.05$ were considered significant. The performance of the non-invasive methods for liver fibrosis was measured as sensitivity, specificity, positive and negative predictive value (PPV and NPV respectively), accuracy, positive and negative likelihood ratio (LR). Sensitivity, specificity, PPV, NPV and accuracy were expressed as percentage. The diagnostic value of the non-invasive biomarkers was expressed and compared using the AUROC and its corresponding 95\% confidence intervals (CI). AUROCs were calculated including non-invasive markers' quantitative values using empirical non parametric method according to DeLong et al. and compared using the method of Hanley et al. ${ }^{38,} 39$

\section{Standardisation of AUROCs according to the prevalence of fibrosis stages}

As recently proposed by Poynard et al., AUROCs were adjusted according to the prevalence of fibrosis stages using the DANA (Difference between advanced and nonadvanced fibrosis). ${ }^{23}$ The DANA is an index for standardising comparisons to transform any different prevalence profile into a homogeneous distribution of fibrosis stages from $\mathrm{F} 0$ to F4, as defined by a prevalence of 0.20 for each of the five METAVIR stages (standard prevalence). DANA was calculated according to the following formula: [(prevalence $\mathrm{F} 2 \times 2+$ prevalence $\mathrm{F} 3 \times 3+$ prevalence $\mathrm{F} 4$ $\times 4) /($ prevalence $\mathrm{F} 2+$ prevalence $\mathrm{F} 3+$ prevalence $\mathrm{F} 4)]$ [prevalence $\mathrm{F} 1 /$ (prevalence $\mathrm{F} 0+$ prevalence F1)]. The adjusted AUROCs (adjAUROCs) were calculated as follows: AdjAUROC = observed AUROC (obAUROC) + $(0.1056) \times(2.5$ - DANA $)$.

\section{RESULTS}

Demographics, laboratory and histological features of the 2411 patients with CLDs

Overall, there were 1391 males and 1020 females with mean age of $46.9 \pm 12.1$ years. Of 1968 patients with CHC, either monoinfected or HIV coinfected, 1381 (70.2\%) were infected with genotype 1 of HCV. Of 253 patients with $\mathrm{CHB}, 13$ (5.1\%) were coinfected with HDV and $46(18.2 \%)$ were $\mathrm{HBeAg}$ positive. The main demographic, laboratory and histological features, and their 


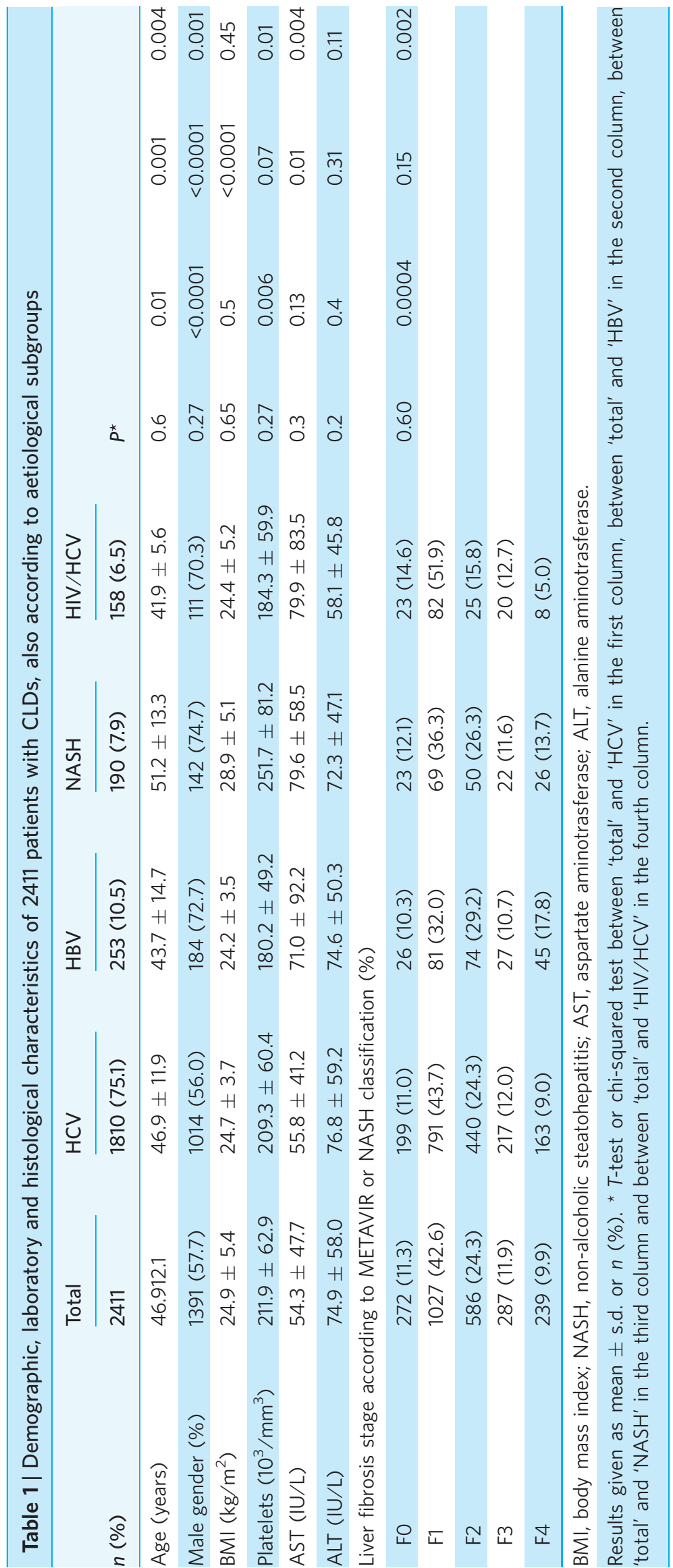




\section{G. Sebastiani et al.}

distribution by aetiological subgroups are summarised in Table 1. Overall, 1112 (46.1\%) patients had significant fibrosis and 239 (9.9\%) had cirrhosis. The mean length of liver specimen was $17.7 \pm 8.4 \mathrm{~mm}$ and the mean number of portal tracts was $10.6 \pm 5.9$. Biopsy length was greater than $15 \mathrm{~mm}$ in $1466(60.8 \%)$ and greater than $20 \mathrm{~mm}$ in 1027 (42.6\%) patients. As compared with the whole population, patients with $\mathrm{CHB}$ and those with $\mathrm{HIV} / \mathrm{HCV}$ coinfection were younger $(P=0.01$ and $P=0.004$ respectively), whereas patients with NASH were significantly older $(P=0.001)$. Male gender was more frequent in $\mathrm{CHB}$ and $\mathrm{HIV} / \mathrm{HCV}$ coinfected patients and in cases with NASH $(P<0.0001, P=0.001$ and $P<0.0001$ respectively). As expected, patients with NASH had a significantly higher BMI when compared with the whole population of the study $(P<0.0001)$. Platelets were significantly lower in patients with $\mathrm{CHB}$ and $\mathrm{HIV} / \mathrm{HCV}$ coinfection with respect to the whole population $(P=0.006$ and $P=0.01$ respectively). Moreover, AST levels were significantly higher in patients with NASH and HIV/HCV coinfection as compared with the whole population $(P=0.01$ and $P=0.004$ respectively). The distribution of liver fibrosis stages also showed some significant differences: patients with CHB had more advanced fibrosis stages, and patients with $\mathrm{HIV} / \mathrm{HCV}$ coinfection had less advanced fibrosis stages when compared with the whole population (signifi- cant fibrosis being present in $57.7 \%$ and $33.5 \%$ of cases vs. $46.1 \% ; P=0.0004$ and $P=0.002$ respectively).

Effect of the stages of hepatic fibrosis on the performance of non-invasive biomarkers to diagnose significant fibrosis

The performance of non-invasive biomarkers to diagnose significant fibrosis and the effect of the stages of hepatic fibrosis are shown in Table 2. Overall, among the noninvasive biomarkers considered, Fibrotest-Fibrosure and APRI showed the best performance. For all non-invasive biomarkers, the correction of the ObAUROC for DANA resulted in a higher AdjAUROC, although this difference was not statistically significant. Fibrotest-Fibrosure was able to classify $100 \%$ of cases, a figure that was significantly higher than that of APRI, Forns' index and Fib-4 $(P<0.0001)$. Even when corrected for DANA, FibrotestFibrosure and APRI showed the best performance for significant fibrosis, as indicated by an AdjAUROC of 0.76 and of 0.74 respectively.

Effect of the stages of hepatic fibrosis on the performance of non-invasive biomarkers to diagnose cirrhosis

The performance of non-invasive biomarkers to diagnose cirrhosis and the effect of the stages of hepatic

Table 2 | Performance of non-invasive biomarkers for significant fibrosis and correction for the stages of hepatic fibrosis in 2411 patients with CLDs

\begin{tabular}{|c|c|c|c|c|c|c|c|}
\hline \multirow[b]{2}{*}{ Cut-off } & \multicolumn{2}{|c|}{ APRI } & \multicolumn{2}{|c|}{ Forns' index } & \multicolumn{2}{|c|}{ Fib-4 } & \multirow{2}{*}{$\begin{array}{l}\begin{array}{l}\text { Fibrotest- } \\
\text { Fibrosure }\end{array} \\
0.49\end{array}$} \\
\hline & 0.5 & 1.5 & 4.2 & 6.9 & 1.45 & 3.25 & \\
\hline Classified cases $(\%)$ & \multicolumn{2}{|c|}{67.5} & \multicolumn{2}{|c|}{56.9} & \multicolumn{2}{|c|}{60.0} & 100 \\
\hline Sensitivity (\%) & 67.9 & 37.4 & 76.0 & 65.6 & 70.5 & 55.0 & 61.0 \\
\hline Specificity (\%) & 71 & 95.9 & 60.0 & 50.8 & 65.0 & 60.0 & 83.0 \\
\hline PPV (\%) & 66.7 & 85.1 & 77.2 & 72.1 & 73.4 & 66.2 & 79.2 \\
\hline NPV (\%) & 72 & 60.6 & 59.3 & 70.4 & 60.1 & 40.5 & 62.4 \\
\hline Accuracy (\%) & 64.3 & 69.5 & 68.5 & 70.9 & 67.3 & 72.0 & 71.3 \\
\hline $\mathrm{LR}+$ & 2.34 & 9.12 & 1.9 & 1.33 & 2.01 & 1.63 & 3.59 \\
\hline LR- & 0.45 & 0.65 & 0.4 & 0.68 & 0.45 & 0.58 & 0.47 \\
\hline DANA & \multicolumn{7}{|c|}{1.90} \\
\hline ObAUROC (95\% Cl) & \multicolumn{2}{|c|}{$0.68(0.62-0.74)$} & \multicolumn{2}{|c|}{$0.67(0.61-0.73)$} & \multicolumn{2}{|c|}{$0.66(0.61-0.71)$} & $0.7(0.65-0.75)$ \\
\hline AdjAUROC (95\% Cl) & \multicolumn{2}{|c|}{$0.74(0.68-0.80)$} & \multicolumn{2}{|c|}{$0.73(0.67-0.79)$} & \multicolumn{2}{|c|}{$0.72(0.67-0.77)$} & $0.76(0.71-0.81)$ \\
\hline
\end{tabular}


fibrosis are shown in Table 3. Overall, among the noninvasive biomarkers considered, APRI and FibrotestFibrosure showed the best performance for cirrhosis. The performance of AAR was rather poor and significantly lower than that of platelets, APRI and FibrotestFibrosure $(P<0.0001)$. APRI and Lok index were able to classify less number of cases than Fibrotest-Fibrosure, AAR and platelets $(86.7 \%$ and $52.3 \%$ vs. $100 \%$ respectively, $P<0.0001)$ For all non-invasive biomarkers, the correction of the ObAUROC for DANA resulted in a higher AdjAUROC, although this difference was not statistically significant. Even when corrected for DANA, APRI and Fibrotest-Fibrosure showed the best performance for cirrhosis, as indicated by an AdjAUROC of 0.80 and of 0.79 respectively. Interestingly, both APRI and Fibrotest-Fibrosure showed a high NPV (>93\%) to exclude cirrhosis.

Impact of aetiology of liver diseases on the performance of non-invasive biomarkers

A subgroup analysis according to different aetiologies was performed for the non-invasive biomarkers and their respective cut-off values that showed the best performance in the whole series of patients. APRI showed its best performance in $\mathrm{CHC}$, with an AdjAUROC of 0.77 for significant fibrosis and an AdjAUROC of 0.83 for cirrhosis (Table 4). Although slightly inferior, the performance of
APRI in $\mathrm{HIV} / \mathrm{HCV}$ coinfected patients was not significantly different from that in monoinfected HCV cases, with an AdjAUROC of 0.74 for significant fibrosis and an AdjAUROC of 0.77 for cirrhosis. In CHB, APRI showed a significantly lower performance than in $\mathrm{CHC}$, with an AdjAUROC of 0.69 for significant fibrosis and an AdjAUROC of 0.66 for cirrhosis $(P<0.0001)$. The performance of APRI was significantly lower for NASH vs. CHC for both significant fibrosis and cirrhosis $(P<0.0001)$. Interestingly, APRI showed a high NPV $(>97 \%)$ to exclude presence of cirrhosis in both $\mathrm{HCV}$ monoinfected and $\mathrm{HIV} / \mathrm{HCV}$ coinfected cases.

Fibrotest-Fibrosure showed its best performance in $\mathrm{CHC}$, with an AdjAUROC of 0.77 for significant fibrosis and 0.79 for cirrhosis (Table 5). The performance of Fibrotest-Fibrosure in $\mathrm{HIV} / \mathrm{HCV}$ coinfected patients was similar to that in $\mathrm{HCV}$ monoinfected cases. In $\mathrm{CHB}$, Fibrotest-Fibrosure showed a slightly reduced performance than in $\mathrm{CHC}$ for diagnosis of cirrhosis, but this difference was not significant. Conversely, performance of Fibrotest-Fibrosure for significant fibrosis was significantly lower for NASH vs. CHC $(P<0.0001)$. On the other hand, the performance of Fibrotest-Fibrosure for cirrhosis was significantly higher in NASH cases vs. HBV cases $(P<0.0001)$. Interestingly, in all aetiologies except for $\mathrm{CHB}$, Fibrotest-Fibrosure showed a very high NPV (>95\%) to exclude presence of cirrhosis.

Table 3 | Performance of non-invasive biomarkers for cirrhosis and correction for the stages of hepatic fibrosis in 2411 patients with CLDs

\begin{tabular}{|c|c|c|c|c|c|c|c|}
\hline \multirow[b]{2}{*}{ Cut-off } & \multirow{2}{*}{$\begin{array}{l}\text { Platelets } \\
150\end{array}$} & \multicolumn{2}{|c|}{ APRI } & \multicolumn{2}{|c|}{ Lok index } & \multirow{2}{*}{$\begin{array}{c}\text { AAR } \\
1\end{array}$} & \multirow{2}{*}{$\begin{array}{l}\text { Fibrotest-Fibrosure } \\
\\
0.75\end{array}$} \\
\hline & & 1 & 2 & 0.2 & 0.5 & & \\
\hline Classified cases (\%) & 100 & \multicolumn{2}{|c|}{86.7} & 52.3 & & 100 & 100 \\
\hline Sensitivity (\%) & 53.3 & 74.0 & 41.3 & 76.0 & 56.5 & 43.4 & 54.1 \\
\hline Specificity (\%) & 85.7 & 82.6 & 93.7 & 40.0 & 60.7 & 58.3 & 90.1 \\
\hline PPV (\%) & 34.6 & 31.7 & 41.8 & 30.5 & 66.0 & 66.7 & 60.8 \\
\hline NPV (\%) & 92.9 & 96.7 & 93.6 & 88.5 & 40.3 & 35 & 93.8 \\
\hline Accuracy (\%) & 77.9 & 81.7 & 88.5 & 66.8 & 69.7 & 48.6 & 86.3 \\
\hline LR+ & 3.72 & 4.25 & 6.56 & 1.27 & 1.44 & 1.04 & 5.46 \\
\hline LR- & 0.54 & 0.31 & 0.63 & 0.6 & 0.71 & 0.97 & 0.51 \\
\hline DANA & & & & & 1.90 & & \\
\hline ObAUROC $(95 \% \mathrm{Cl})$ & $0.72(0.66-0.78)$ & 0.74 & $0.69-0.79)$ & 0.63 & $0.57-0.69)$ & $0.54(0.48-0.60)$ & $0.73(0.68-0.78)$ \\
\hline AdjAUROC $(95 \% \mathrm{Cl})$ & $0.78(0.72-0.84)$ & 0.80 & $0.75-0.85)$ & 0.69 & $0.63-0.75)$ & $0.6(0.54-0.66)$ & $0.79(0.74-0.84)$ \\
\hline
\end{tabular}

AdjAUROC, adjusted area under the curve; APRI, AST-to-platelet ratio index; Cl, confidence interval; DANA, difference between advanced and non-advanced fibrosis; LR, likelihood ratio; NPV, negative predictive value; ObAUROC, observed area under the curve; PPV, positive predictive value. 


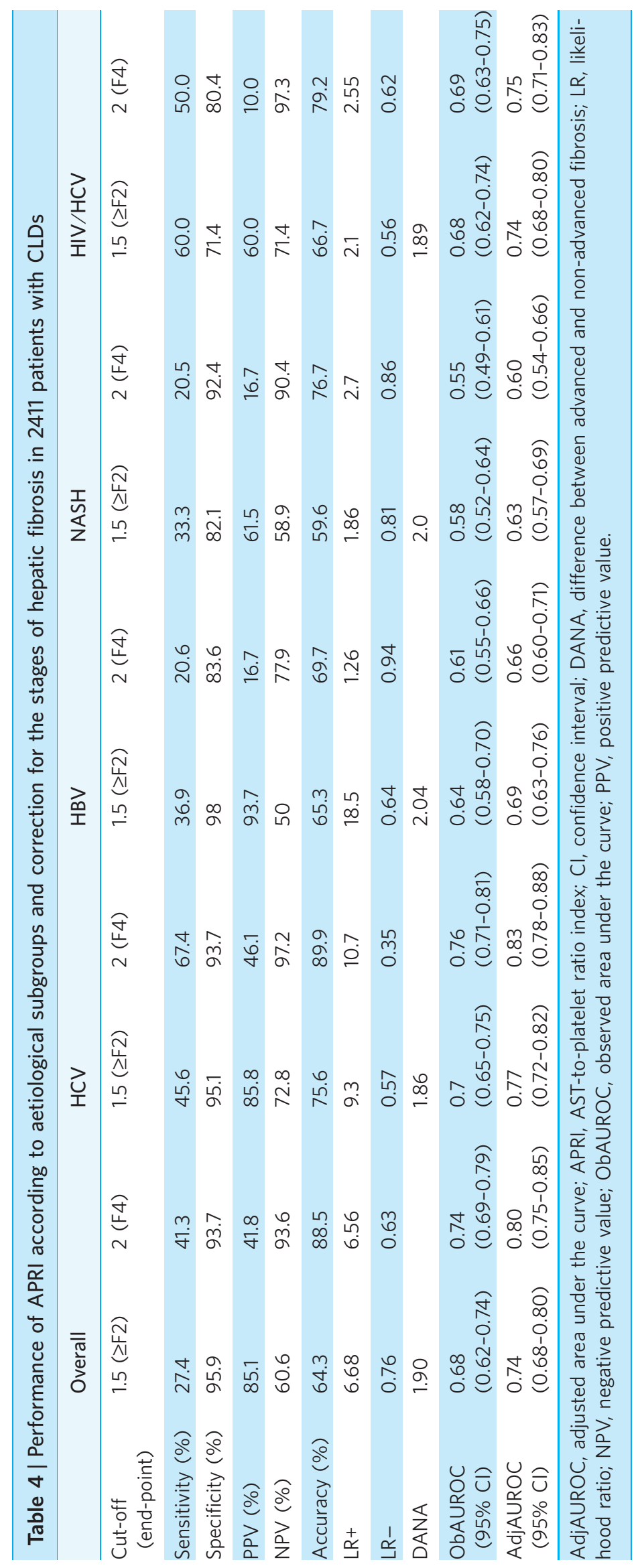


Liver fibrosis biomarkers, aetiology and fibrosis stages

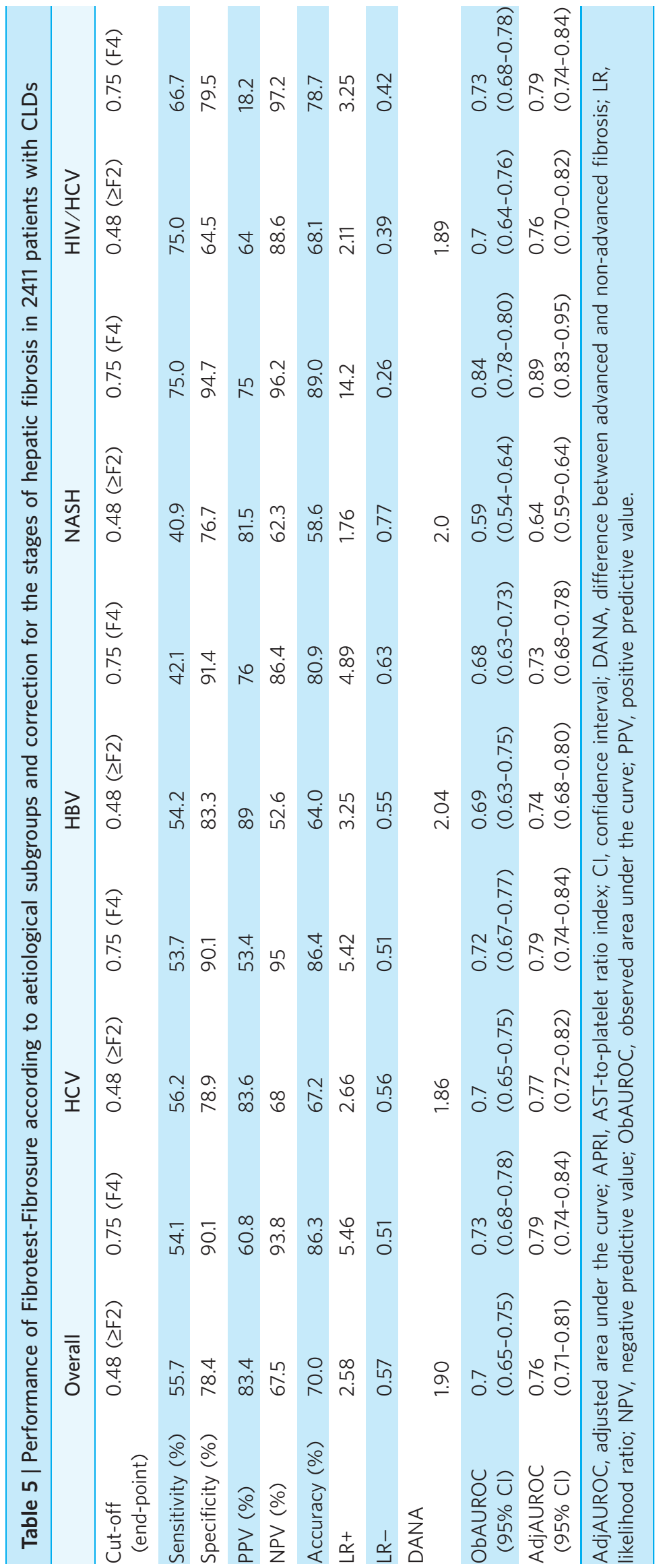




\section{G. Sebastiani et al.}

Effect of other variables on the performance of non-invasive biomarkers

The influence of other variables on the performance of the non-invasive biomarkers, which exhibited the best performance, was also investigated. Inter-centre variability was marginal (data not shown). The effect of liver biopsy size on the performance of non-invasive biomarkers was also studied. As regards APRI, similar performance was observed for subjects with biopsy samples $<20 \mathrm{~mm}$ as compared with those with specimens $>20 \mathrm{~mm}: \quad$ AdjAUROC $=0.72(0.66-0.78,95 \%$ CI $)$ vs. $0.75(0.70-0.80,95 \% \mathrm{CI})$ for significant fibrosis, and 0.79 $(0.75-0.83,95 \%$ CI $)$ vs. $0.80(0.75-0.85,95 \%$ CI $)$ for cirrhosis respectively. On the same line, as regards Fibrotest-Fibrosure, similar performance was observed for subjects with biopsy samples $<20 \mathrm{~mm}$ as compared with those with specimens $>20 \mathrm{~mm}$ : AdjAUROC $=0.74$ $(0.68-0.80,95 \% \mathrm{CI})$ vs. $0.76(0.71-0.81,95 \% \mathrm{CI})$ for significant fibrosis, and $0.79(0.75-0.83,95 \% \mathrm{CI})$ vs. 0.79 (0.74-0.84, 95\% CI) for cirrhosis respectively.

\section{Performance of non-invasive biomarkers in $\mathrm{HCV}$ patients with normal ALT and in elderly patients with $\mathrm{CHC}$}

To assess the performance of non-invasive biomarkers and the effect of DANA in patients who may have a peculiar distribution of liver fibrosis stages, a dedicated analysis was conducted on two subgroups of patients: (i) HCV patients with normal ALT, who are expected to have a lower prevalence of advanced fibrosis stages; (ii) HCV elderly patients (age $\geq 65$ years), who are expected to have a higher prevalence of advanced fibrosis stages. Indeed, these two subgroups of patients represent the vast majority of $\mathrm{HCV}$ carriers in the general population in most Western Countries, with an indication to liver biopsy that is often debated and controversial. ${ }^{40-42}$

Overall, 595 (32.9\%) HCV cases had normal ALT and $190(10.5 \%)$ cases were over 65 years of age. The main demographic, laboratory and histological features of the two subgroups and comparison with the whole population study are summarised in Table S1. When compared with the whole study population, patients with normal ALT were significantly younger $(P=0.001)$ and had a lower prevalence of advanced fibrosis stages (significant fibrosis being present in $29.5 \%$ vs. $45.3 \%, P<0.0001$ ). Moreover, they present with lower frequency of male gender $(P<0.0001)$ and higher platelets $(P<0.0001)$ with respect to the whole population.

Overall, performance of all non-invasive biomarkers for significant fibrosis in patients with normal ALT was rather poor, with ObAUROC $<0.65$ for all the non-inva- sive markers tested (Table S2). When correction for DANA was applied, APRI and Fibrotest-Fibrosure showed the highest performance, which remained, however, only discrete (AdjAUROC of 0.71 for APRI and 0.70 for Fibrotest-Fibrosure). These figures were lower than those observed in the whole population, even if the difference was not statistically significant. On the same line, performance of all non-invasive biomarkers for cirrhosis was lower than in the whole population, the highest ObAUROC being 0.65 (Table S3). The performance of AAR was significantly lower than that of platelets, APRI and Fibrotest-Fibrosure $(P<0.0001)$. When correction for DANA was applied, APRI and Fibrotest-Fibrosure showed the highest performance, with an AdjAUROC of 0.73 for both markers.

As regards elderly patients with $\mathrm{HCV}$, they had a significantly lower prevalence of male gender $(P<0.0001)$, higher BMI $(P=0.04)$, lower platelets $(P=0.005)$ and higher AST and ALT $(P=0.01$ and $P<0.0001$ respectively) (Table S1). As expected, elderly HCV patients present with a higher prevalence of advanced fibrosis stages (significant fibrosis being present in $71.0 \%$ vs. $45.3 \%$, $P<0.0001)$ when compared with the whole population.

Overall, performance of all non-invasive biomarkers for significant fibrosis in HCV elderly patients was similar to that of the whole population (Table S4). Among the non-invasive biomarkers considered, Fibrotest-Fibrosure and APRI showed the best performance for diagnosis of significant fibrosis, as indicated by an AdjAUROC of 0.74 for both markers. As regards the diagnosis of cirrhosis, platelets showed the best performance, as indicated by an AdjAUROC of 0.87. Interestingly, platelets showed the highest NPV (>95\%) to exclude cirrhosis (Table S5).

\section{DISCUSSION}

The results of this large-scale, multicentre, independent study showed that aetiology of CLDs is a major factor influencing the performance of a series of non-invasive biomarkers. Moreover, APRI and Fibrotest-Fibrosure had the best performance for the diagnosis of significant fibrosis and cirrhosis in various aetiologies of CLDs. This study represents one of the few and the largest in which a direct comparison of the performance of non-invasive biomarkers for liver fibrosis in aetiologies other than HCV has been carried out. Our study has also shown that the performance of non-invasive biomarkers for liver fibrosis may be distinct in special subgroups of patients who are expected to have a peculiar distribution of fibrosis stages, such as HCV carriers with normal ALT and elderly HCV patients. Although these two 
subgroups of HCV patients are highly represented in the general population and the indication to liver biopsy remains controversial, few studies have specifically investigated the performance of non-invasive biomarkers in them. Moreover, our study represents the first largescale, multicentre, independent study that evaluated the effect of the stages of hepatic fibrosis on the performance of the most validated non-invasive biomarkers for liver fibrosis. Indeed, it has been suggested that the prevalence of liver fibrosis stages may be a major factor of variability in assessing the diagnostic value of a fibrosis marker, usually evaluated and compared using AUROC, and it may be a cause of unsatisfactory results. ${ }^{23}$ According to our results, the prevalence of liver fibrosis stages may have an effect on the AUROC, and should be taken into account. Indeed, in our experience, the AUROC was higher of 0.04-0.08 for all non-invasive biomarkers studied when corrected for DANA.

Staging of liver fibrosis has been recognised as of paramount importance for both prognosis and management of CLDs. ${ }^{3,4}, 36,43$ Liver biopsy has always been regarded as the reference standard for liver fibrosis assessment. ${ }^{10}$ However, it is invasive, costly and not feasible as screening tool for liver fibrosis in CLDs, which affect millions of people worldwide. ${ }^{11,44}$ In recent years, many efforts have been dedicated towards the identification of noninvasive tools for a more rapid, practical and less expensive initial screening for disease stage and risk of progression. Several non-invasive biomarkers for liver fibrosis have been described, including simple methods with virtually no cost, such as platelets, AAR, APRI, Fib4, Lok index and Forns' index, and more elaborated patented panels of biomarkers, such as Fibrotest-Fibrosure. ${ }^{15-21}$ In clinical practice, the implementation of noninvasive biomarkers for liver fibrosis is still limited by the scepticism shared by many clinicians on their performance in substitution of liver histology, especially in aetiologies other than $\mathrm{CHC}$, where limited validation exists. $^{12,13,22,45}$ Among the non-invasive biomarkers investigated in our study, APRI and Fibrotest-Fibrosure showed the best performance for diagnosis of significant fibrosis and cirrhosis in all aetiologies of CLDs, even when the AUROC was corrected for DANA. This finding is coherent with other studies that compared the performance of non-invasive biomarkers for liver fibrosis. ${ }^{27}, 46-49$ Importantly, we showed that performance of APRI and Fibrotest-Fibrosure may significantly differ among various aetiologies of CLDs. As regards APRI, it showed its best performance in $\mathrm{HCV}$ monoinfected patients. Although the performance was slightly inferior, no sig- nificant difference was observed in $\mathrm{HIV} / \mathrm{HCV}$ coinfected cases. Moreover, we found a very high NPV (>97\%) with a cut-off value of 2 to exclude cirrhosis in both monoinfected and coinfected cases that could be useful in clinical practice to select patients in whom the risk of having cirrhosis is marginal. It has been suggested that performance of APRI may be diminished in coinfected patients because of HIV-related or antiretroviral-related thrombocytopenia. ${ }^{50-52}$ Conversely, our results are in line with a recent meta-analysis, which reported a tendency for lower performance of APRI in $\mathrm{HIV} / \mathrm{HCV}$ coinfected patients that did not reach the statistical significance. ${ }^{51}$ On the same line, other studies reported a similar performance of APRI in HIV/HCV coinfected patients. ${ }^{53,54}$ In $\mathrm{CHB}$, the performance of APRI was somehow unsatisfactory for both significant fibrosis and cirrhosis, with an AUROC always $<0.70$, even when corrected for DANA. These results are in line with previous studies. ${ }^{27,} 55$ Only one study reported a good performance of APRI for significant fibrosis, as indicated by an AUROC of $0.86 .{ }^{56}$ The differences between the patient populations in this study could be responsible for this disagreement. As regards NASH, the performance of APRI was poor for both significant fibrosis and cirrhosis, as indicated by an AdjAUROC of 0.63 and 0.60 respectively. Similar results have been reported in a small-scale study. ${ }^{57}$ Another study investigated the performance of APRI for prediction of cirrhosis in only 50 patients. The authors found a discrete performance, as indicated by an AUROC of 0.786 for cirrhosis. ${ }^{58}$ Our results, based on 190 consecutive patients, could not confirm this initial report.

As regards Fibrotest-Fibrosure, it showed a good performance in all aetiologies of CLDs, except for the diagnosis of significant fibrosis in NASH. Moreover, in all aetiologies except for CHB, Fibrotest-Fibrosure showed a very high NPV (>95\%) to exclude presence of cirrhosis, a result that could be useful in clinical practice to select patients in whom the risk of having cirrhosis is marginal. Similar to APRI, Fibrotest-Fibrosure showed its best performance in HCV monoinfected cases. The performance of Fibrotest-Fibrosure in patients with HIV/HCV coinfection was similar to that found for monoinfected $\mathrm{HCV}$ patients. One initial study of $130 \mathrm{HIV} / \mathrm{HCV}$ coinfected patients coming from the group that patented the test reported an excellent AUROC of 0.856 for the prediction of significant fibrosis. ${ }^{29}$ However, a following study of 272 patients, which took into account also DANA, reported lower performances that were similar to our results, as indicated by an ObAUROC of 0.64 for significant fibrosis, that raised to 0.78 when corrected by 


\section{G. Sebastiani et al.}

DANA, and 0.81 for cirrhosis. ${ }^{52}$ The performance of Fibrotest-Fibrosure in CHB was investigated in few studies. The reported AUROC ranged from 0.76 to 0.85 for diagnosis of significant fibrosis and from 0.76 to 0.80 for diagnosis of cirrhosis that are somehow in line with the results of the present study. ${ }^{27,}{ }^{28}$ Interestingly, it has been recently suggested that Fibrotest-Fibrosure may have a role in the follow-up of HBV inactive carriers as well as better selection of patients who require a liver biopsy. ${ }^{33,59}$ As regards NASH, very few reports investigated the role of Fibrotest-Fibrosure. ${ }^{60}$ In our experience, Fibrotest-Fibrosure had a high performance to diagnose cirrhosis, with a very high NPV (>96\%) to exclude cirrhosis and an overall AdjAUROC of 0.89. As, to our knowledge, this is the first independent application of Fibrotest-Fibrosure in NASH patients, this result deserves attention and should be confirmed by other studies. It should be here underlined that Fibrotest-Fibrosure has the advantage of classifying all the cases, while APRI leaves up to $41 \%$ of cases unclassified.

Thanks to the large number of patients included in our cohort, we were also able to perform a subgroup analysis aimed at assessing the performance of non-invasive biomarkers and the effect of DANA in patients who may have a peculiar distribution of liver fibrosis stages, including $\mathrm{HCV}$ patients with normal ALT and HCV elderly patients. In clinical practice, these groups of patients are very frequent and may be the ones in whom liver biopsy is even more questionable, and a reliable non-invasive tool is highly needed. ${ }^{40,}{ }^{42}$ Indeed, regardless of ALT levels, the decision to initiate anti-viral therapy should be individualised based on the severity of liver disease assessed by liver biopsy, the potential of serious side effects, the likelihood of response and the presence of comorbidities. ${ }^{40}$ Few studies have investigated the role of non-invasive biomarkers in patients with normal ALT. ${ }^{61-66}$ Some studies suggested that performance of non-invasive biomarkers is in line with that in patients with elevated ALT. ${ }^{61}$ Conversely, other reports suggested that performance of non-invasive biomarkers may be somewhat reduced in patients with normal ALT. ${ }^{62,63,65,66}$ Our study, based on a large series of cases in which the prevalence of significant fibrosis was in line with previous population studies, ${ }^{14}$ indicates that the performance of non-invasive biomarkers is reduced in patients with normal ALT, even when AUROC is adjusted according to fibrosis stages distribution. Consequently, the clinical use and interpretation of these markers in this subgroup of patients should be cautious. In elderly $\mathrm{HCV}$ patients, the greater prevalence of liver disease is linked to higher morbidity and mortality, which are excellent reasons for improving the evaluation of liver fibrosis and to better identify indication to antiviral therapies. ${ }^{41,} 42$ A single report by Poynard and colleagues investigated the role of Fibrotest-Fibrosure in elderly patients with $\mathrm{CHC}$, and a good performance of the patented panel was reported. ${ }^{67}$ Interestingly, in our study, platelets showed a very good performance to diagnose cirrhosis in this subgroup of patients. Indeed, a cutoff of $150000 / \mathrm{mm}^{3}$ had an excellent NPV to exclude the presence of cirrhosis. A good performance of platelets in excluding cirrhosis has been already reported, but this is the first study to assess its performance in a large series of elderly HCV patients. ${ }^{68}$ This finding could be useful in clinical practice as first line approach through a simple, inexpensive biomarker to select elderly patients in whom the risk of having cirrhosis is trivial. Elderly $\mathrm{HCV}$ patients are indeed more at risk of having advanced fibrosis, but the clinician is less prone to perform a liver biopsy in them as the procedure is likely to be more risky than in younger patients. ${ }^{41}$ It has been shown that complications related to liver biopsy procedure are more severe in patients with underlying cardiac or pulmonary disease, who also have a higher operative risk than younger patients. ${ }^{69}$ As a consequence of the fear of the physician towards liver biopsy, elderly patients may be at risk of undermanagement, even though they generally present with a more severe disease and a faster fibrosis progression.

The present study has several strengths. The use of a multicentre cohort of well-characterised patients from numerous centres across the world gave us the opportunity to obtain a very large population of patients of different aetiologies, and with a wide spectrum of liver disease, ranging from absence of fibrosis to well-compensated, subclinical cirrhosis. For many of the non-invasive biomarkers investigated, this study represents one of the few independent validations, particularly in aetiologies other than CHC.

We acknowledge several limitations to our analysis. Indeed, this is a retrospective study and potential selection bias of patients may derive. The mean length of the liver biopsy in this study was $17.7 \mathrm{~mm}$, which is somehow suboptimal according to recently recommended threshold of $20 \mathrm{~mm} .{ }^{10}$ However, we have investigated the effect of liver biopsy size on the performance of non-invasive markers and we found a similar performance for subjects with biopsy samples of $<20 \mathrm{~mm}$ as compared to those with specimens $>20 \mathrm{~mm}$. This result is in line with other reports, in which the length of liver biopsy did not 
influence significantly the performance of the non-invasive methods investigated. ${ }^{19,} 70$ Interestingly, according to other recommendations, a biopsy specimen of $15 \mathrm{~mm}$ length gives sufficient histological information. ${ }^{71}$ No information about fragmentation of liver biopsy specimens was available in the present study. Indeed, it has been demonstrated that the number of fragments may have a significant impact on the AUROC of non-invasive markers. ${ }^{72}$ Our study did not have a central pathologist for the interpretation of liver histology. Lack of evaluation by a single Pathologist of all biopsies could be seen as a weakness of our study, but it better describes what occurs in real life. Moreover, the use of morphometric quantification of liver fibrosis would have minimised subjectivity in the assessment of hepatic fibrosis. ${ }^{73,74}$ It should be here underlined that liver biopsy remains an imperfect gold standard due to intraobserver and interobserver variability, which mainly depends on the quality of liver specimen. ${ }^{75-78}$ Moreover, it has been shown that even in the subpopulation with biopsy length $\geq 25 \mathrm{~mm}$, there is still $35 \%$ of risk of false positive/negative of the biopsy result. $^{76}$ Mehta and colleagues have recently suggested that in the most favourable scenario, an AUROC $>0.90$ cannot be achieved when assessing significant fibrosis even for a perfect markers and that a correct way to validate non-invasive markers for liver fibrosis vs. liver biopsy would be to perform prospective studies using clinical outcomes. ${ }^{79}$ Nevertheless, as liver biopsy remains the only direct way to assess liver histology, guidelines still recommend it for the staging of hepatic fibrosis. $3,36,43$

In conclusion, this large-scale, independent study suggests that the performance of non-invasive biomarkers can be highly influenced by aetiology of CLD. The stages of hepatic fibrosis may also influence the performance as expressed by AUROC, especially in subgroups of patients where peculiar distribution of liver fibrosis stages is expected. Finally, among a series of non-invasive biomarkers, APRI and Fibrotest-Fibrosure showed the best performance. Both these non-invasive biomarkers may be used in clinical practice to safely exclude cirrhosis in $\mathrm{HCV}$ monoinfected and HIV/HCV coinfected cases. The choice of the method may depend on the local availability and experience of the clinician. APRI could be the first choice in regions with limited healthcare resources. On the other hand, Fibrotest-Fibrosure has the advantage to classify all cases, while APRI leaves a significant percentage of cases unclassified. In patients with NASH, Fibrotest-Fibrosure may also be applied in clinical practice to exclude cirrhosis, while it cannot be satisfactorily used for the diagnosis of significant fibrosis. In elderly
HCV patients, presence of cirrhosis may be excluded with high certainty with a specific cut-off of platelets. For the diagnosis of significant fibrosis, especially in $\mathrm{HCV}$ carriers with normal ALT, performance of non-invasive biomarkers is not such to substitute liver biopsy. Further prospective studies about application of the most promising non-invasive biomarkers for liver fibrosis, especially in aetiologies other than HCV and using clinical outcomes as reference standard, are needed.

\section{ACKNOWLEDGEMENTS}

Declaration of personal interests: GS has served as a speaker and consultant for Istituto Biochimico Italiano (IBI) and MSD, and has received research funding from Roche. LC has served as a speaker for Echosens and Ferrer. PH has served as a speaker for MSD and has received research funding from Roche. $\mathrm{PH}$ owns patent: PCT protease inhibitors. SP has served as a speaker, a consultant and an advisory board member for GSK, BMS, Boehringer Ingelheim, Tibotec/Janssen Cilag, Gilead, Roche and Schering-Plough, and has received research funding from BMS, Gilead, Roche and Merck/ Schering-Plough. AM has served as a speaker, a consultant and an advisory board member for Jannssen, MSD and Roche, and has received research funding from MSD and Roche. VD has served as a speaker, a consultant and an advisory board member for Roche, Gilead, BMS, MSD and Dohme. MP has served as a speaker for Bayer, Bristol-Myers-Squibb, Gilead Sciences, Merck and Roche. MV has nothing to declare. MB has served as a speaker and consultant for Roche, Schering-Plough, MSD, BMS, Gilead, GSK and Vertex. AA has served as a speaker and consultant for Roche, Gilead, Novartis, BMS, J\&J, MSD and Schering-Plough, and has received research funding from Gilead, MSD and BMS. Declaration of funding interests: None.

\section{SUPPORTING INFORMATION}

Additional Supporting Information may be found in the online version of this article:

Table S1. Demographic, laboratory and histological characteristics of $595 \mathrm{HCV}$ patients with normal ALT and of $190 \mathrm{HCV}$ patients with age $\geq 65$ years and comparison with the whole HCV population.

Table S2. Performance of non-invasive biomarkers for significant fibrosis and correction for the stages of hepatic fibrosis in $595 \mathrm{HCV}$ cases with normal ALT.

Table S3. Performance of non-invasive biomarkers for cirrhosis and correction for the stages of hepatic fibrosis in $595 \mathrm{HCV}$ cases with normal ALT. 


\section{G. Sebastiani et al.}

Table S4. Performance of non-invasive biomarkers for significant fibrosis and correction for the stages of hepatic fibrosis in 190 HCV elderly cases.

Table S5. Performance of non-invasive biomarkers for cirrhosis and correction for the stages of hepatic fibrosis in $190 \mathrm{HCV}$ elderly cases.
Please note: Wiley-Blackwell are not responsible for the content or functionality of any supporting materials supplied by the authors. Any queries (other than missing material) should be directed to the corresponding author for the article.

\section{REFERENCES}

1. Anonymous. Global surveillance and control of hepatitis C. Report of a WHO Consultation organized in collaboration with the Viral Hepatitis Prevention Board, Antwerp, Belgium. J Viral Hepat 1999; 6: 35-47.

2. Angulo P. Nonalcoholic fatty liver disease. N Engl J Med 2002; 346: 1221-31.

3. Sorrell MF, Belongia EA, Costa J, et al. National Institutes of Health Consensus Development Conference Statement: management of hepatitis B. Ann Intern Med 2009; 150: 104-10.

4. Alberti A, Clumeck N, Collins S, et al. Short statement of the first European Consensus Conference on the treatment of chronic hepatitis B and C in HIV co-infected patients. J Hepatol 2005; 42 : 615-24.

5. Sulkowski MS, Thomas DL. Hepatitis C in the HIV-infected person. Ann Intern Med 2003; 138: 197-207.

6. Friedman SL. Liver fibrosis - from bench to bedside. J Hepatol 2003; 38(Suppl. 1): S38-53.

7. Alberti A, Chemello L, Benvegnu L. Natural history of hepatitis C. J Hepatol 1999; 31(Suppl. 1): 17-24.

8. de Franchis R, Hadengue A, Lau G, et al. EASL International Consensus Conference on Hepatitis B. 13-14 September, 2002 Geneva, Switzerland. Consensus statement (long version). J Hepatol 2003; 39(Suppl. 1): S3-25.

9. Clark JM, Diehl AM. Nonalcoholic fatty liver disease: an underrecognized cause of cryptogenic cirrhosis. JAMA 2003; 289: 3000-4.

10. Rockey DC, Caldwell SH, Goodman ZD, Nelson RC, Smith AD. Liver biopsy. Hepatology 2009; 49: 1017-44.

11. Wong JB, Koff RS. Watchful waiting with periodic liver biopsy versus immediate empirical therapy for histologically mild chronic hepatitis C. A cost-effectiveness analysis. Ann Intern Med 2000; 133: 665-75.

12. Sebastiani G, Alberti A. Non invasive fibrosis biomarkers reduce but not substitute the need for liver biopsy. World J Gastroenterol 2006; 12: 3682-94.
13. Manning DS, Afdhal NH. Diagnosis and quantitation of fibrosis. Gastroenterology 2008; 134: 1670-81.

14. Alberti A, Noventa F, Benvegnu L, Boccato S, Gatta A. Prevalence of liver disease in a population of asymptomatic persons with hepatitis $\mathrm{C}$ virus infection. Ann Intern Med 2002; 137: 961-4.

15. Lackner C, Struber G, Bankuti C, Bauer B, Stauber RE. Noninvasive diagnosis of cirrhosis in chronic hepatitis $\mathrm{C}$ based on standard laboratory tests. Hepatology 2006; 43: 378-9.

16. Wai CT, Greenson JK, Fontana RJ, et al. A simple noninvasive index can predict both significant fibrosis and cirrhosis in patients with chronic hepatitis C. Hepatology 2003; 38: 518-26.

17. Forns X, Ampurdanes S, Llovet JM, et al. Identification of chronic hepatitis $\mathrm{C}$ patients without hepatic fibrosis by a simple predictive model. Hepatology 2002; 36: 986-92.

18. Vallet-Pichard A, Mallet V, Nalpas B, et al. FIB-4: an inexpensive and accurate marker of fibrosis in HCV infection. Comparison with liver biopsy and fibrotest. Hepatology 2007; 46: 32-6.

19. Lok AS, Ghany MG, Goodman ZD, et al. Predicting cirrhosis in patients with hepatitis $\mathrm{C}$ based on standard laboratory tests: results of the HALT-C cohort. Hepatology 2005; 42: 282-92.

20. Giannini E, Risso D, Botta F, et al. Validity and clinical utility of the aspartate aminotransferase-alanine aminotransferase ratio in assessing disease severity and prognosis in patients with hepatitis $\mathrm{C}$ virus-related chronic liver disease. Arch Intern Med 2003; 163: 218-24.

21. Imbert-Bismut F, Ratziu V, Pieroni L, Charlotte F, Benhamou Y, Poynard T. Biochemical markers of liver fibrosis in patients with hepatitis $C$ virus infection: a prospective study. Lancet 2001; 357: 1069-75.

22. Smith JO, Sterling RK. Systematic review: non-invasive methods of fibrosis analysis in chronic hepatitis C. Aliment Pharmacol Ther 2009; 30: 557-76.

23. Poynard T, Halfon P, Castera L, et al. Standardization of ROC curve areas for diagnostic evaluation of liver fibrosis markers based on prevalences of fibrosis stages. Clin Chem 2007; 53: 1615-22.

24. Shaheen AA, Myers RP. Diagnostic accuracy of the aspartate aminotransferaseto-platelet ratio index for the prediction of hepatitis C-related fibrosis: a systematic review. Hepatology 2007; 46: 912-21.

25. Kleiner DE, Brunt EM, Van Natta M, et al. Design and validation of a histological scoring system for nonalcoholic fatty liver disease. Hepatology 2005; 41: 1313-21.

26. The French METAVIR Cooperative Study Group. Intraobserver and interobserver variations in liver biopsy interpretation in patients with chronic hepatitis C. Hepatology 1994; 20: 15-20.

27. Sebastiani G, Vario A, Guido M, Alberti A. Sequential algorithms combining non-invasive markers and biopsy for the assessment of liver fibrosis in chronic hepatitis B. World J Gastroenterol 2007; 13: $525-31$.

28. Myers RP, Tainturier MH, Ratziu V, et al. Prediction of liver histological lesions with biochemical markers in patients with chronic hepatitis B. J Hepatol 2003; 39: 222-30.

29. Myers RP, Benhamou Y, Imbert-Bismut $\mathrm{F}$, et al. Serum biochemical markers accurately predict liver fibrosis in HIV and hepatitis $\mathrm{C}$ virus co-infected patients. AIDS 2003; 17: 721-5.

30. Palmeri ML, Wang MH, Rouze NC, et al. Noninvasive evaluation of hepatic fibrosis using acoustic radiation forcebased shear stiffness in patients with nonalcoholic fatty liver disease. $J$ Hepatol 2011; 55: 666-72.

31. Mendeni M, Foca E, Gotti D, et al. Evaluation of liver fibrosis: concordance analysis between noninvasive scores (APRI and FIB-4) evolution and predictors in a cohort of HIV-infected patients without hepatitis $\mathrm{C}$ and $\mathrm{B}$ infection. Clin Infect Dis 2011; 52: 1164-73.

32. Macias J, Giron-Gonzalez JA, GonzalezSerrano M, et al. Prediction of liver fibrosis in human immunodeficiency virus/hepatitis $\mathrm{C}$ virus coinfected patients by simple non-invasive indexes. Gut 2006; 55: 409-14. 
33. Castera L, Bernard PH, Le Bail B, et al. Transient elastography and biomarkers for liver fibrosis assessment and followup of inactive hepatitis B carriers. Aliment Pharmacol Ther 2011; 33: 45565.

34. Practice guidelines for the treatment of hepatitis C: recommendations from an AISF/SIMIT/SIMAST Expert Opinion Meeting. Dig Liver Dis 2010; 42: 81-91.

35. European Association For The Study Of The L. EASL Clinical Practice Guidelines: management of chronic hepatitis B. J Hepatol 2009; 50: 227-42.

36. Ghany MG, Strader DB, Thomas DL, Seeff LB. Diagnosis, management, and treatment of hepatitis C: an update. Hepatology 2009; 49: 1335-74.

37. Argo CK, Northup PG, Al-Osaimi AM, Caldwell SH. Systematic review of risk factors for fibrosis progression in nonalcoholic steatohepatitis. J Hepatol 2009; 51: 371-9.

38. DeLong ER, DeLong DM, Clarke-Pearson DL. Comparing the areas under two or more correlated receiver operating characteristic curves: a nonparametric approach. Biometrics 1988; 44: 837-45.

39. Hanley JA, McNeil BJ. The meaning and use of the area under a receiver operating characteristic (ROC) curve. Radiology 1982; 143: 29-36.

40. Calvaruso V, Craxi A. Implication of normal liver enzymes in liver disease. $J$ Viral Hepat 2009; 16: 529-36.

41. Wachtel MS, Zhang Y, Kaye KE, Chiriva-Internati M, Frezza EE. Increased age, male gender, and cirrhosis, but not steatosis or a positive viral serology, negatively impact the life expectancy of patients who undergo liver biopsy. Dig Dis Sci 2007; 52: 2276-81.

42. Floreani A. Liver diseases in the elderly: an update. Dig Dis 2007; 25: 138-43.

43. Ratziu V, Bellentani S, Cortez-Pinto H, Day C, Marchesini G. A position statement on NAFLD/NASH based on the EASL 2009 special conference. J Hepatol 2010; 53: 372-84.

44. Gilmore IT, Burroughs A, Murray-Lyon IM, Williams R, Jenkins D, Hopkins A. Indications, methods, and outcomes of percutaneous liver biopsy in England and Wales: an audit by the British Society of Gastroenterology and the Royal College of Physicians of London. Gut 1995; 36: 437-41.

45. Martinez SM, Crespo G, Navasa M, Forns X. Noninvasive assessment of liver fibrosis. Hepatology 2011; 53: 325-35.

46. Sebastiani G, Vario A, Guido M, et al. Stepwise combination algorithms of non-invasive markers to diagnose significant fibrosis in chronic hepatitis C. J Hepatol 2006; 44: 686-93.

47. Bourliere M, Penaranda G, Renou C, et al. Validation and comparison of indexes for fibrosis and cirrhosis prediction in chronic hepatitis $\mathrm{C}$ patients: proposal for a pragmatic approach classification without liver biopsies. J Viral Hepat 2006; 13: 659-70.

48. Halfon P, Bourliere M, Deydier R, et al. Independent prospective multicenter validation of biochemical markers (fibrotest-actitest) for the prediction of liver fibrosis and activity in patients with chronic hepatitis C: the fibropaca study. Am J Gastroenterol 2006; 101: 547-55.

49. Leroy V, Hilleret MN, Sturm N, et al. Prospective comparison of six non-invasive scores for the diagnosis of liver fibrosis in chronic hepatitis C. J Hepatol 2007; 46: 775-82.

50. Scaradavou A. HIV-related thrombocytopenia. Blood Rev 2002; 16: 73-6.

51. Lin ZH, Xin YN, Dong QJ, et al. Performance of the aspartate aminotransferaseto-platelet ratio index for the staging of hepatitis C-related fibrosis: an updated meta-analysis. Hepatology 2011; 53: 72636.

52. Cacoub P, Carrat F, Bedossa P, et al. Comparison of non-invasive liver fibrosis biomarkers in HIV/HCV co-infected patients: the fibrovic study - ANRS HC02. J Hepatol 2008; 48: 765-73.

53. Nunes D, Fleming C, Offner G, et al. HIV infection does not affect the performance of noninvasive markers of fibrosis for the diagnosis of hepatitis $\mathrm{C}$ virusrelated liver disease. J Acquir Immune Defic Syndr 2005; 40: 538-44.

54. Shaheen AA, Myers RP. Systematic review and meta-analysis of the diagnostic accuracy of fibrosis marker panels in patients with HIV/hepatitis C coinfection. HIV Clin Trials 2008; 9: 43-51.

55. Wai CT, Cheng CL, Wee A, et al. Noninvasive models for predicting histology in patients with chronic hepatitis $B$. Liver Int 2006; 26: 666-72.

56. Shin WG, Park SH, Jang MK, et al. Aspartate aminotransferase to platelet ratio index (APRI) can predict liver fibrosis in chronic hepatitis B. Dig Liver Dis 2008; 40: 267-74.

57. Loaeza-del-Castillo A, Paz-Pineda F, Oviedo-Cardenas E, Sanchez-Avila F, Vargas-Vorackova F. AST to platelet ratio index (APRI) for the noninvasive evaluation of liver fibrosis. Ann Hepatol 2008; 7: 350-7.

58. Fujii H, Enomoto M, Fukushima W, et al. Noninvasive laboratory tests proposed for predicting cirrhosis in patients with chronic hepatitis $\mathrm{C}$ are also useful in patients with non-alcoholic steatohepatitis. J Gastroenterol 2009; 44: 60814.

59. Ngo Y, Benhamou Y, Thibault V, et al. An accurate definition of the status of inactive hepatitis $\mathrm{B}$ virus carrier by a combination of biomarkers (FibroTest-
ActiTest) and viral load. PLoS ONE 2008; 3: e2573.

60. Ratziu V, Massard J, Charlotte F, et al. Diagnostic value of biochemical markers (FibroTest-FibroSURE) for the prediction of liver fibrosis in patients with nonalcoholic fatty liver disease. BMC Gastroenterol 2006; 6: 6.

61. Poynard T, Munteanu M, Ngo Y, et al. Diagnostic value of FibroTest with normal serum aminotransferases. Hepatology 2006; 43: 374-5.

62. Liu CH, Lin JW, Tsai FC, et al. Noninvasive tests for the prediction of significant hepatic fibrosis in hepatitis $C$ virus carriers with persistently normal alanine aminotransferases. Liver Int 2006; 26: 1087-94.

63. Fabris C, Smirne C, Toniutto P, et al. Assessment of liver fibrosis progression in patients with chronic hepatitis $\mathrm{C}$ and normal alanine aminotransferase values: the role of AST to the platelet ratio index. Clin Biochem 2006; 39 : 339-43.

64. Poynard T, Munteanu M, Ngo Y, et al. FibroTest is effective in patients with normal transaminases, when accuracy is standardized on fibrosis stage prevalence. J Viral Hepat 2008; 15: 472-3.

65. Sebastiani G, Vario A, Guido M, Alberti A. Performance of noninvasive markers for liver fibrosis is reduced in chronic hepatitis $\mathrm{C}$ with normal transaminases. J Viral Hepat 2008; 15: 212-8.

66. Colletta C, Smirne C, Fabris C, et al. Value of two noninvasive methods to detect progression of fibrosis among $\mathrm{HCV}$ carriers with normal aminotransferases. Hepatology 2005; 42: 838-45.

67. Thabut D, Le Calvez S, Thibault V, et al. Hepatitis $\mathrm{C}$ in 6,865 patients $65 \mathrm{yr}$ or older: a severe and neglected curable disease? Am J Gastroenterol 2006; 101: 1260-7.

68. Castera L, Le Bail B, Roudot-Thoraval F, et al. Early detection in routine clinical practice of cirrhosis and oesophageal varices in chronic hepatitis C: comparison of transient elastography (FibroScan) with standard laboratory tests and noninvasive scores. J Hepatol 2009; 50: 5968.

69. McGill DB, Rakela J, Zinsmeister AR, Ott BJ. A 21-year experience with major hemorrhage after percutaneous liver biopsy. Gastroenterology 1990; 99: 1396-400.

70. Guido M, Alberti A, Bellati G, et al. Fibrotest vs. liver biopsy: a multicenter, independent evaluation of performance. Hepatology 2007; 46 [abstract].

71. Shiha G, Sarin SK, Ibrahim AE, et al. Liver fibrosis: consensus recommendations of the Asian Pacific Association for the Study of the Liver (APASL). Hepatol Int 2009; 3: 323-33. 


\section{G. Sebastiani et al.}

72. Poynard T, Halfon P, Castera L, et al. Variability of the area under the receiver operating characteristic curves in the diagnostic evaluation of liver fibrosis markers: impact of biopsy length and fragmentation. Aliment Pharmacol Ther 2007; 25: 733-9.

73. Masseroli M, Caballero T, O’Valle F, Del Moral RM, Perez-Milena A, Del Moral RG. Automatic quantification of liver fibrosis: design and validation of a new image analysis method: comparison with semi-quantitative indexes of fibrosis. $J$ Hepatol 2000; 32: 453-64.
74. Huss S, Schmitz J, Goltz D, Fischer HP, Buttner R, Weiskirchen R. Development and evaluation of an open source Delphi-based software for morphometric quantification of liver fibrosis. Fibrogenesis Tissue Repair 2010; 3: 10.

75. Colloredo G, Guido M, Sonzogni A, Leandro G. Impact of liver biopsy size on histological evaluation of chronic viral hepatitis: the smaller the sample, the milder the disease. J Hepatol 2003; 39: 239-44.

76. Bedossa P, Dargere D, Paradis V. Sampling variability of liver fibrosis in chronic hepatitis C. Hepatology 2003; 38: 1449-57.

77. Guido M, Rugge M. Liver biopsy sampling in chronic viral hepatitis. Semin Liver Dis 2004; 24: 89-97.

78. Rousselet MC, Michalak S, Dupre F, et al. Sources of variability in histological scoring of chronic viral hepatitis. Нepatology 2005; 41: 257-64.

79. Mehta SH, Lau B, Afdhal NH, Thomas DL. Exceeding the limits of liver histology markers. J Hepatol 2009; 50: 36-41. 